\title{
qt cm 0.1.2: a Python implementation of the Neelin-Zeng Quasi-Equilibrium Tropical Circulation Model
}

\author{
J. W.-B. Lin \\ Physics Department, North Park University, 3225 W. Foster Ave., Chicago, Illinois 60625, USA
}

Received: 23 September 2008 - Published in Geosci. Model Dev. Discuss.: 30 October 2008

Revised: 2 February 2009 - Accepted: 2 February 2009 - Published: 11 February 2009

\begin{abstract}
Historically, climate models have been developed incrementally and in compiled languages like Fortran. While the use of legacy compiled languages results in fast, time-tested code, the resulting model is limited in its modularity and cannot take advantage of functionality available with modern computer languages. Here we describe an effort at using the open-source, object-oriented language Python to create more flexible climate models: the package qt cm, a Python implementation of the intermediate-level Neelin-Zeng Quasi-Equilibrium Tropical Circulation model (QTCM1) of the atmosphere. The qt cm package retains the core numerics of QTCM1, written in Fortran to optimize model performance, but uses Python structures and utilities to wrap the QTCM1 Fortran routines and manage model execution. The resulting "mixed language" modeling package allows order and choice of subroutine execution to be altered at run time, and model analysis and visualization to be integrated in interactively with model execution at run time. This flexibility facilitates more complex scientific analysis using less complex code than would be possible using traditional languages alone, and provides tools to transform the traditional "formulate hypothesis $\rightarrow$ write and test code $\rightarrow$ run model $\rightarrow$ analyze results" sequence into a feedback loop that can be executed automatically by the computer.
\end{abstract}

\section{Introduction}

Although early weather and climate models, beginning with Richardson's "Forecast Factory" in 1922 (Edwards, 2000), led the development of the field of scientific computing, over the past few decades, climate models have not, in general, kept up with advances in computing languages and struc-

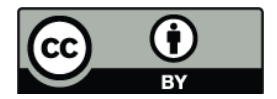

Correspondence to: J. W.-B. Lin (jlin@ northpark.edu) tures. Many climate models are still written in compiled languages (primarily Fortran), and utilize the same programming structures familiar to a Fortran programmer of the 1970's. On the positive side, this continued reliance on Fortran results in very fast code that runs on almost all platforms, the ability to reuse legacy code, and the availability of welltested libraries, which have been optimized over decades of use.

At the same time, the continued development of climate models in Fortran has made it difficult to utilize programming language advances that increase the modularity and robustness of scientific code. Being mainly a procedural language, Fortran has traditionally lacked the default programming structures to organize a model into truly self-contained units, thus limiting modularity. Fortran subroutine function calls may utilize long and unwieldy argument lists, its default variables are not self-describing, and variables exist in a loosely controlled namespace; this can result in brittle code where undetectable errors easily propagate. Finally, as a compiled language, Fortran is non-interactive and requires separate compiling and linking steps. This hinders informal small-scale testing, prevents users from interacting with the model at run time, and can result in a longer development cycle. Recent versions of Fortran (e.g., Fortran 95, 2003) have added some of these modern features to the language, but scientific programs, in general, make limited use of these new features.

Modern computer languages have constructs that overcome many of these difficulties, though at a penalty in performance. These languages possess the tools to manage the variable namespace that older procedural languages lack, and thus modern languages can avoid lengthy hard-wired argument lists through the use of dictionaries and the creation of specialty data structures and classes that ensure the right variables are available and used when needed. Modern objectoriented frameworks bind metadata to variables, as well as the functions that act on the variables. Such contextualized

Published by Copernicus Publications on behalf of the European Geosciences Union. 
variables make possible additional levels of modular decomposition. Object-oriented programming can also produce code of higher quality (e.g., Johnson, 2002), that more closely emulates real-world entities (e.g., Pennington et al., 1995). Some modern languages are also interpreted; in those languages, source code is directly executed at run time without separate compiling and linking steps, thereby enabling interactive debugging and execution.

One such modern language is Python (van Rossum, 2008), an interpreted, object-oriented, multi-platform, open-source language used in a variety of software applications, including as a robust scientific computing platform (Oliphant, 2007). In climate studies, Python has been used as the core language for data analysis (e.g., PCMDI, 2006), visualization (e.g., Hunter and Dale, 2007), and modeling (e.g., PyCCSM, 2008). Python's object-orientation and higher-level data structures and tools (e.g., dictionaries, string and file utilities) permits numerous ways of decomposing a model into modular units. Its extensive suite of higher-level analysis tools (e.g., statistics, visualization), accessible at run time, enables modeling and analysis to occur concurrently. As an interpreted language, Python's lack of a separate lengthy compile step greatly simplifies debugging and testing, and permits changes in the program to be made at run time.

While it is more difficult to write robust code in compiled languages, the code is usually very fast. Modern languages, however, while producing much more robust and stable code, exact a cost in performance. Naturally, we want the best of both worlds, both speed and simplicity: "mixed language" environments (Oliphant, 2007) are a solution. In such an environment, the user-interface and calling infrastructure of the model is written in a modern language while the performance sensitive code is written in a compiled language. A wrapper generator automatically creates extension modules (as shared object libraries) of the compiled language modules, making them accessible to the modern language. A number of wrapper generator packages exist for Python, including f2py (Peterson, 2005) which wraps Fortran modules, and SWIG (Beazley, 1997) which wraps C/C++ code.

In the present work, we describe a Python implementation of an intermediate-level atmospheric circulation model originally written in Fortran. By wrapping the Fortran code within a Python object structure, the package, qt $\mathrm{cm}$, provides a modular and interactive model where the user can alter order and choice of subroutine execution, and analyze and visualize model results, all dynamically at run time. The result is a climate modeling environment that can transform parts of the "formulate hypothesis $\rightarrow$ write and test code $\rightarrow$ run model $\rightarrow$ analyze results" sequence into a feedback loop that can be executed automatically by the computer.

Section 2 briefly describes the Neelin-Zeng QuasiEquilibrium Tropical Circulation Model (QTCM1). In Sect. 3, we describe the construction of a Python implementation of QTCM1, the qt cm package. Section 4 gives examples of the use of the qt cm package, which illustrate the benefits of a mixed language environment for climate modeling. We finish with discussion and conclusions in Sect. 5.

\section{The Neelin-Zeng QTCM1}

The QTCM1 is a primitive equation-based intermediate-level atmospheric model that focuses on simulating the tropical atmosphere (Neelin et al., 2002). Being more complicated than a simple model, the model retains full non-linearity with a basic representation of baroclinic instability, includes a radiative-convective feedback package, and includes a simple land soil moisture routine (but does not include topography). The QTCM1 has been used in a variety of studies, including investigations of Madden-Julian oscillation maintenance mechanisms (Lin et al., 2000), stochastic convective parameterization (Lin and Neelin, 2000, 2002), El NiñoSouthern Oscillation teleconnection patterns (Gushchina et al., 2006), and vegetation-atmosphere interactions (Zeng et al., 1999).

QTCM1 differs from most full-scale general circulation models (GCMs) in that the vertical temperature, humidity, and velocity structures of the atmosphere are represented by a truncated Galerkin expansion in the vertical, instead of finite-differenced pressure levels. The vertical basis functions of the expansion are chosen based on analytical solutions under convective quasi-equilibrium conditions, and thus in the tropics, where convective quasi-equilibrium effects dominate, the solution is asymptotically exact. Away from the tropics, the model behaves as a two-layer model. In principle, a Galerkin model can have any number of baroclinic basis functions accompanying its barotropic basis: QTCM1 has a single baroclinic mode, and hence the " 1 " in its name. In the horizontal, the model discretizes the domain using a staggered Arakawa C-grid (Mesinger and Arakawa, 1976) and a default resolution of $5.625^{\circ}$ longitude by $3.75^{\circ}$ latitude.

By using tailored vertical profiles, the QTCM1 delivers reasonable simulations of the tropics at a fraction of the computational cost of a full-scale GCM. Its relative simplicity also makes it far easier to diagnose than a full-scale GCM, potentially resulting in greater understanding and comprehension of model results. Neelin and Zeng (2000) presents a comprehensive description of the model's formulation, and Zeng et al. (2000) describes the model's climatology. A detailed manual (Neelin et al., 2002) describes the structure of the Fortran code. Neelin and Zeng (2000) is based upon v2.0 of QTCM1 and Zeng et al. (2000) is based on QTCM1 v2.1. The qt cm package is based on QTCM1 v2.3.

\section{The Python qt cm package}

The qtcm package is an implementation of QTCM1 in a Python-based object-oriented modeling framework, using f2py to create extension module versions of the Fortran 
modules (as shared object libraries). At the package home page (http://www.johnny-lin.com/py_pkgs/qtem/), the full source code and a comprehensive user's guide is available for download. The User's Guide and source code for the version of the model described in the present work is available as a supplement at http://www.geosci-model-dev.net/2/1/2009/ gmd-2-1-2009-supplement.zip, and are covered by licenses separate from the present work. The User's Guide (Lin, 2008) provides detailed information regarding installing, using, troubleshooting, and adding code to the package. In the present work, we provide an overview of qt $\mathrm{cm}$ 's structure and function. Parts of this section are copied and/or adapted from Lin (2008).

\subsection{Object-oriented programming}

Because Python is an object-oriented language, the fundamental programming unit is not the subroutine, but instead is the "object". In a procedural language, data and functions that operate on data are two separate entities. In an objectoriented language, these two entities are bound together in a single construct, the object. Because of this framework, functions are automatically considered in context with the data they operate on, and vice versa. This lessens the risk of errors that occur when data is manipulated by functions that were never intended to be used on that kind of data.

Data bound to an object are called "attributes" of that object, and functions that operate on that data are called "methods" of that object. In Python, the attributes of an object are specified by a name that comes after a period at the end of the object name. Thus, model.runname refers to the runname attribute of the model object. Methods are similarly named; however, to call a method, a parameter list (even if empty) must be specified. Thus, model.run_session() calls the run_session method bound to the model object.

In general, Python objects consist of two types of attributes and methods: public and private. Public attributes and methods are accessible to the general user. Private attributes and methods, on the other hand, are designed to be accessed only by developers. In Python, private attributes and methods have names prepended by one or two underscores.

Objects are created from a "template" that defines the attributes and methods that go into that object. The template is known as a "class," and individual objects that are derived from a class are called "instances" of that class. Creating an object that is an instance of a class is known as "instantiating" the object. In the example above, model is an instance of the Qtcm class, which defines the runname attribute and run_session method. There is no limit to the number of instances of a class, and all instances of a class have access to the attributes and methods defined by the class.

Python's highest level of organization is the package, a library of related modules. Modules, in Python, are individual files that define related objects, functions, and variables, and thus a package is a directory of module files. A single module can contain an unlimited number of objects, functions, and variables.

\subsection{Package and model structure}

The qt $\mathrm{cm}$ package consists of two shared object libraries and four main submodules. The two shared object libraries are compiled and generated by $\mathrm{f} 2 \mathrm{py}$ during package installation, and will not need to be recompiled prior to model execution. The defaults submodule defines various defaults for the model, the field submodule defines class Field (key model variables and parameters are instances of this class), the plot submodule defines routines used for quick visualization of model results, and the qt $\mathrm{cm}$ submodule defines the class Qt cm (which defines model objects).

A model in the qt cm package is defined as an instance of the class Qt $\mathrm{cm}$. Because the qt $\mathrm{cm}$ package wraps Fortran routines with a Python layer, there are two types of variables associated with Qt $\mathrm{cm}$ model instances: those defined at the Python-level and those defined at the Fortranlevel. Some variables, while defined separately at both the Python and Fortran levels (i.e., they do not share the same memory space), have the same names and functions in both levels of the model. Those variables are known as "field variables" and are considered to be defined at both the Pythonlevel and Fortran-level (an example of such a variable is QC, the precipitation). Qt $\mathrm{cm}$ instances have public methods (get_qtcm1_item and set_qt cm1_item) for passing the values of field variables back-and-forth between the Python and Fortran levels.

All field variables and most model parameters (such as time step, input and output directory names, etc.) are instances of the Field class. A Field instance stores the value of the variable in an attribute named value, and metadata (e.g., units, long name, etc.) related to the variable as other instance attributes. If the value of the field variable is an array, the value stored in the attribute value is a NumPy (van der Walt, 2008) array. Only the value of a Field instance can be passed to its Fortran counterpart (when it exists), because standard Fortran variables cannot hold metadata.

All model parameters (e.g., time step, etc.) are attributes of Qt $\mathrm{cm}$ instances. Field variables, at the Python-level, are also Qt cm instance attributes. Model parameter and field variable values can be passed into the model instance on instantiation via the input keyword parameter list, or set after instantiation by changing the instance attribute. If these parameters and variables are not set manually, they are set to default values given in the defaults submodule. Table 1 lists the key instance attributes and methods for the Field and Qt $\mathrm{cm}$ classes. 
Table 1. Key public instance attributes and methods for Field and Qt cm instances. Note that for Qt cm instances, field variables are also attributes, with attribute names corresponding to the ids of the fields.

\begin{tabular}{|c|c|c|}
\hline Class & Type & Name and Description \\
\hline Field & Attributes & $\begin{array}{l}\text { id: A string naming the model parameter or field variable (e.g., "Qc", "mrestart"). } \\
\text { value: The value of the field. } \\
\text { units: A string giving the units of the field. } \\
\text { long_name: A string giving a description of the field. } \\
\text { rank: Returns the rank of value. } \\
\text { typecode: Returns the typecode of value. }\end{array}$ \\
\hline Qtcm & Attributes & $\begin{array}{l}\text { compiled_form: Describes the form of the compiled Fortran version of the QTCM1 model. } \\
\text { coupling_day: Current value of the atmosphere-ocean coupling day. } \\
\text { init_with_instance_state: Initialize run session with the Qtcm model instance state. } \\
\text { runlists: Lists of methods and other run lists that can be executed by the run_list method. } \\
\text { sodir: Name of temporary directory containing shared object files for this Qtcm instance. } \\
\text { get_qtcm1_item: Get field from the compiled QTCM1 model. } \\
\text { make_snapshot: Make copy of the current state of the run session's variables. } \\
\text { plotm: Plot mean output for a given model field. } \\
\text { qtcm: Run the atmosphere over a coupling interval step. } \\
\text { run_list: Run run list(s) and/or instance methods. } \\
\text { run_session: Run a model run session. } \\
\text { set_qtcm1_item: Set Python-accessible compiled QTCM1 model fields. } \\
\text { sync_set_py_values_to_snapshot: Set Python attributes to a previous make_snapshot output. } \\
\text { varinit: Initialize model variables in a run session. }\end{array}$ \\
\hline
\end{tabular}

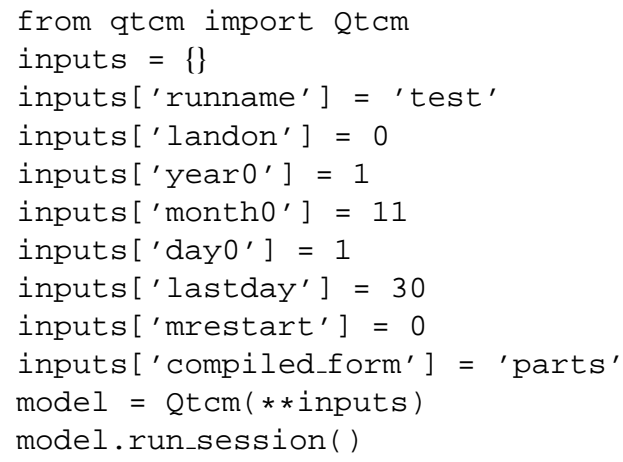

Fig. 1. A simple qt cm run.

\subsection{Creating a model instance and running the model}

Figure 1 shows a simple example of a model instance being created and run. Model instances are created using standard Python syntax; in Fig. 1, model = Qtcm ( $* \star$ inputs) creates a model instance model. In this example, we make use of a feature in Python where keyword parameter argument lists can be passed in as a dictionary (a set of key/value pairs), where the dictionary's keys correspond to the names of the keyword parameters, and the associated value in the dictionary corresponds to the input value of the keyword parameter; the variable inputs is such a dictionary. Based on the values of inputs shown in Fig. 1, the model instance is configured to make an aquaplanet run (set by landon), starting from November 1 , Year 1 (set by year 0 , month 0 , and day0), running for 30 days (set by lastday) from a newly initialized model state (set by mrestart). The model's netCDF (Unidata, 2007) output filenames will contain the string given by runname. By default, the model uses climatological sea-surface temperatures (SST) for the lower-boundary forcing over the ocean.

The keyword compiled_form defines which of the two types of Fortran extension modules, derived from the Fortran QTCM1 code, the model instance will link to. The first type permits very little control over the compiled Fortran routines at the Python level, and is selected by setting compiled_form='full'. The second allows a user, from the Python-level, to control model execution in the Fortran-level all the way down to the atmospheric timestep level. This extension module is selected by setting compiled_form $=$ 'parts'. In general, most users will set compiled_form = 'part $\mathrm{s}^{\prime}$, and thus we assume this setting for the rest of the present work. See Lin (2008) for details about this keyword.

Once the model has been instantiated, running the model requires just a call of the run_session method. In most cases, no input parameters need to be passed at this call. In Fig. 1, this is given in the last line. At the beginning and end of the run_session call, the values of all field variables at the Python and Fortran levels are synchronized to match each other. 


\subsection{Run sessions}

Once we instantiate and configure a model instance, we can use the instance for any number of runs. We call each of these runs using the same model instance a "run session." In a run session, the model is run from day 1 of simulation to the day specified by the lastday attribute. A run session is a "complete" model run, at the beginning of which all Fortranlevel field variables are set to the values given at the Pythonlevel, and at the end of which restart files are written, the values at the Python-level are overwritten by the values from the Fortran-level, and a Python-accessible snapshot is taken of the model variables that were written to the restart file.

Before and after a run session, model variables are easily accessed from the Python level, and can be changed at will just by changing the value of the pertinent model instance attribute. The new values can then be used at the next run session of the model instance. To continue a second run session after an initial run session, set the keyword parameter cont in the input list of a run_session method call.

Figure 2 gives an example of two run sessions, where the second run session is a continuation of the first, and with changes made to a field variable between the two run sessions. The first run session lasts 10 days, and is given by the setting of the lastday keyword parameter. Between these run sessions, the value of field variable $u 1$ (the zonal wind associated with the first baroclinic mode) is doubled, and this doubled value is used in the second run session. The second run session lasts 30 days.

The change between the two run sessions in the simple example given in Fig. 2 is uninteresting, but the example illustrates how the qt cm Python framework opens up possibilities of interactive analysis with the model. Because Python is an interpreted language, the code in Fig. 2 does not have to be written in a file, compiled, linked, and executed; the code can be typed in during run time. Between run sessions, we can conduct and visualize more complex analyses of the model, and use the results of those analyses to change the model configuration for the next run session. And since Python is a complete programming language, we can also automate these analyses, without leaving the modeling environment. The important benefits of this feature are described in Sect. 4.

\subsection{Passing restart snapshots between run sessions}

Sometimes, we want to branch a number of model runs from the same starting point. The QTCM1 writes restart files for that purpose, and a $Q t \mathrm{~cm}$ instance can also make use of those files by setting the mrestart attribute accordingly. This restart mechanism is straightforward to use, but becomes difficult to manage when many restart files are involved.

The qt $\mathrm{cm}$ package provides a way to take, store, and apply restart snapshots at the Python-level, by storing a snapshot as a dictionary. At the end of a run session, a snap-

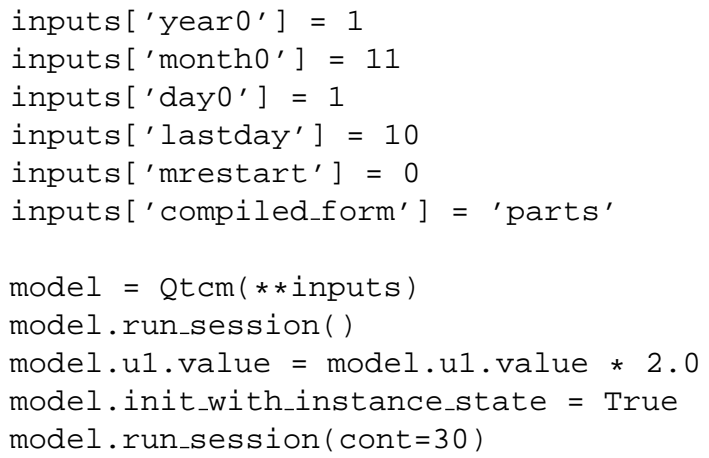

Fig. 2. An example of two qt $\mathrm{cm}$ run sessions where the second run session is a continuation of the first. Assume inputs is a dictionary as in Fig. 1, and that earlier in the script the run name and all input and output directory names were added to the dictionary.

shot of the model state is automatically taken and stored as the instance attribute snapshot. The snapshot includes the date of the model and prognostic variables like T1. You can store this attribute as another Python variable for later use. Figure 3 shows an example of saving the model snapshot as the variable mysnapshot, and using that snapshot to initialize a later run session. The method sync_set_py_values_to_snapshot initializes the model to the values of mysnapshot, and setting the attribute init_with_instance_state to True prior to calling run_session the second time will force the model to use the current instance state as the run session's initial values.

\subsection{Creating multiple models}

Creating multiple QTCM1 Fortran models requires maintaining and operating on different sets of source code, as well as compiling each set of source code separately to obtain the desired multiple executables. With the qt $\mathrm{cm}$ package, creating multiple models is as easy as instantiating multiple $Q t \mathrm{~cm}$ instances. For instance, to create two Python QTCM1 models, model1 and model2, just enter in the following:

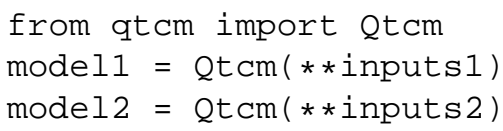

where inputs 1 and inputs 2 are separate dictionaries specifying the input keyword parameters. Recall that nearly any model variable or parameter can, in principle, be set via input keyword parameters. Thus, inputs 1 and inputs 2 could be different in the number of days the model is integrated, whether the land scheme is on or off, the initial values of the prognostic variables, etc. modell and model 2 do not have any variables in common, including in the extension modules holding the Fortran code, and thus the two 
model.run_session()

mysnapshot $=$ model. snapshot

model.sync_set_py_values_to_snapshot (snapshot=mysnapshot)

model.init_with_instance_state = True

model.run_session()

Fig. 3. An example of using a snapshot from one qt $\mathrm{cm}$ run session as the restart for a second run session.

model.run_session()

mysnapshot $=$ model. snapshot

model1. sync_set_py_values_to_snapshot ( napshot=mysnapshot)

model2 . sync_set_py_values_to_snapshot ( napshot=mysnapshot)

model1.run_session ()

model2.run_session()

Fig. 4. An example of using a snapshot from one $q t \mathrm{~cm}$ run session as the restart for run sessions in multiple other model instances.

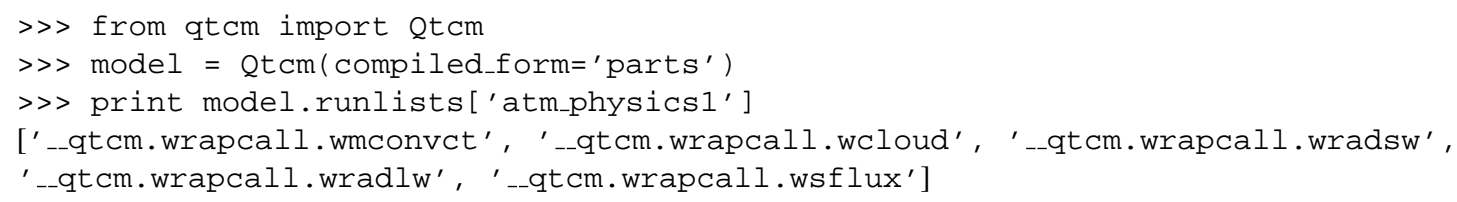

Fig. 5. Contents of run list 'atmphysics $1^{\prime}$, the set of routines to execute to calculate atmospheric physics at one instant in time, as displayed during a Python interpreter session.

instances are two truly independent models. Each instance automatically links to a separate copy of the extension modules, which are saved in temporary directories.

\subsection{Passing restart snapshots between multiple models}

In Sect. 3.5, we saw how a model snapshot can be saved to a separate variable and used to initialize a later run session. Of course, since mysnapshot is an independent dictionary, we are not limited to using it only with the model instance the snapshot originally came from. Figure 4 shows an example of using a snapshot to initialize run sessions in multiple models.

\subsection{Run lists}

Of all the features the Python infrastructure enables us to create in our wrapping of the QTCM1 model, run lists may be the most valuable. A run list in the qt cm package is a Python list that specifies a series of Python or Fortran methods, functions, subroutines (or other run lists) that will be executed when the list is passed into a call of the Qt $\mathrm{cm}$ instance method run_list. Since routines in run lists are identified by strings (instead of, for instance, as a memory pointer to a library archive object file), and Python lists are mutable, run lists are fully changeable at run time. As a result, what routines the model executes are also fully changeable at run time.
Run lists are stored in a dictionary set to the $Q t \mathrm{~cm}$ instance attribute runlists. The dictionary key for the run list's entry is the run list name. Figure 5 shows an interactive Python session that prints out the contents of run list 'atm_physics1'. This run list specifies the set of routines used to calculate atmospheric physics at one instant in time. Each entry of the list is a string and refers to the name of the wrapped Fortran routine that calculates moist convection, cloud effects, shortwave radiative flux, longwave radiative flux, and surface fluxes, respectively.

To change the order of the calculation, or to add, delete, or replace the routines being called, just change the elements of the list using any of the list methods provided by Python (e.g., append). For instance, to reorder the run list in Fig. 5 so that the convection scheme is called after all the other physics schemes, type in:

$$
\begin{aligned}
& \text { tmp }=\text { model.runlists ['atm_physics1'] } \cdot \text { pop (0) } \\
& \text { model.runlists['atm_physics1'] .append (tmp) }
\end{aligned}
$$

In Fig. 5, all the routines given in the run list are Fortran subroutines and require no parameters to be passed in via an argument list. Run lists can, however, specify Python functions and methods and other run lists. For both Python and Fortran routines, the run list feature can also accommodate routines that have argument lists. Figure 6 shows the run list for initializing the atmospheric portion of the model. The first two routines executed by the run list are Fortran subroutines without any input parameters. The third is the Qtcm 


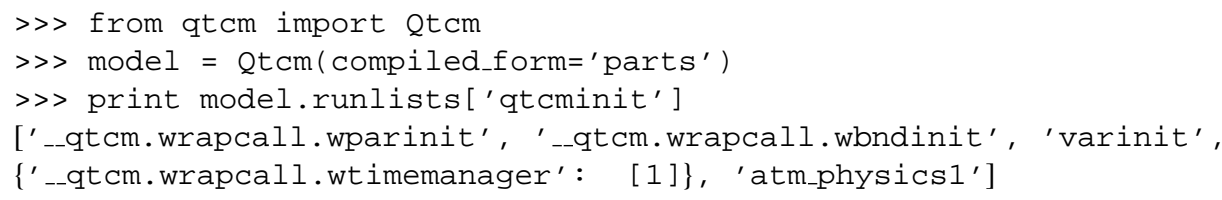

Fig. 6. Contents of run list 'qtcminit', the set of routines to execute to initialize the atmospheric portion of the model, as displayed during a Python interpreter session.

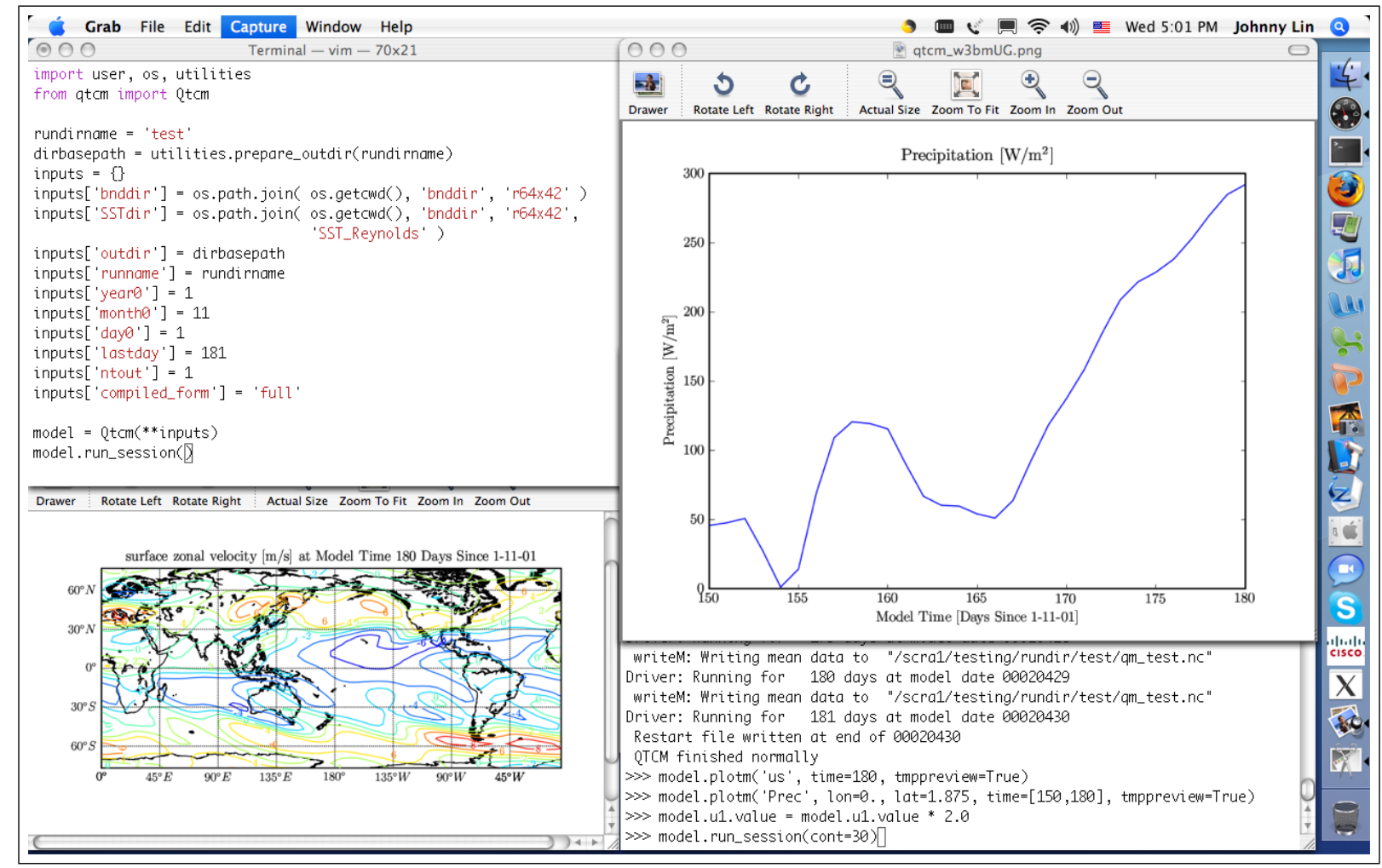

Fig. 7. Screenshot of an interactive modeling session using the qt cm package. The upper-left panel shows the source code file specifying the run. The lower-right panel shows the Python interpreter session making the run. The two plot windows display the plots generated by the plotm calls from the Python interpreter command line.

instance method varinit, also without input parameters in the calling argument list. The fourth element of the run list is a Fortran subroutine, but with one input parameter in its calling argument list. The final routine is not a routine at all, but another run list. Regardless of what kind of routine or run list is specified, the syntax is still the same: a string or a one-element dictionary with a string as the key. Lin (2008) gives details about run lists.

\subsection{Output, visualization, and analysis}

The Qt cm model instance writes instantaneous and mean output to netCDF files. The netCDF data format is a platform independent binary format that permits metadata to be saved with the data. There are a number of packages for
Python that can read and manipulate netCDF data, such as the Climate Data Analysis Tools (PCMDI, 2006).

The Matplotlib package (Hunter and Dale, 2007) for Python generates 1-D and 2-D plots using Matlab-like syntax. Qt cm instances have a method plotm which reads the netCDF output files and uses Matplotlib to create line or contour plots of user-specified slices of the data. Figure 7 shows an interactive modeling session with the qt cm package where the user has created visualizations of a variety of parameters at run time.

Because the $\mathrm{gtcm}$ package makes the Fortran-level variables accessible from the Python level, the user can use any analysis tools at the Python-level on data from those Fortranlevel variables, in addition to the netCDF output, and send the values as desired back to the Fortran-level, all during run 
Table 2. Wall-clock times (sec) for the average of three 365 day aquaplanet runs using climatological sea surface temperature as the lower boundary forcing (Lin, 2008). All runs are executed as single threads. The "Pure" column refers to runs using the pure-Fortran QTCM1, while "Wrap" refers to the Python wrapped qt cm package (v0.1.1) with compiled_form ='part $s$ '.

\begin{tabular}{lcc}
\hline System & Pure & Wrap \\
\hline Mac OS X: MacBook 1.83 GHz Intel & 152.59 & 158.94 \\
Core Duo running Mac OS X 10.4.10. & & \\
& & \\
Ubuntu GNU/Linux: Dell PowerEdge & 43.73 & 47.45 \\
860 with 2.66 GHz Quad Core In- & & \\
tel Xeon processors (64 bit) running & & \\
Ubuntu 8.04.1 LTS. & & \\
\hline
\end{tabular}

time. This enables the user to utilize the powerful analysis tools provided by the Climate Data Analysis Tools, SciPy (van der Walt, 2008), and other Python packages, during as well as after run time.

\subsection{Model performance}

Because the model's core numerics are written in Fortran, with Python providing a sophisticated programmer/userinterface, the performance penalty of the qtcm package (with compiled_form =' parts'), compared to the pure-Fortran QTCM1 is approximately 4-9\% (the penalty for compiled_form =' full' is less). Table 2 gives wall-clock values for qt $\mathrm{cm}$ running on two platforms, Mac OS X and Ubuntu GNU/Linux.

\section{Example uses of the qt cm package}

By wrapping the Fortran QTCM1 with a Python layer, the qt $\mathrm{cm}$ package permits us to accomplish science tasks that would otherwise require a labyrinthine set of shell scripts, temporary input and output files, and source code versions. In this section, we describe a few such science tasks to illustrate what the Python wrapping buys us. The examples in this section are taken from Lin (2008).

\subsection{Conditionally explore parameter space}

Figure 8 provides an example of code that explores different values of mixed-layer depth ( $\mathrm{ziml}$ ) over a set of 30 day runs, as a function of maximum zonal wind associated with the first baroclinic mode (u1) magnitude, until it finds a case where the maximum of $u 1$ is greater than $10 \mathrm{~m} / \mathrm{s}$. (The relationship between ziml and the maximum of the speed of $u 1$, where $\operatorname{ziml}=0.1 * \operatorname{maxu} 1$, is made up.) With each iteration, the new run uses the snapshot from a previous run as its initialization (as well as the new value of ziml); the try statement is used to ensure the model works even if mysnapshot is not defined (which is the case the first time around).

If we implemented this science task using the pure-Fortran QTCM1 and shell scripts, we would probably have to write a separate program (possibly in a separate data analysis language like IDL, Matlab, or NCL) to analyze model output. Required parameters might be passed through an operating system pipe, or through namelists and temporary files. Automating modeling with analysis in such an environment can be difficult, limited, and error prone. The qt $\mathrm{cm}$ package allows us to take advantage of Python's numerical computing capabilities so that we can embed our traverse of parameter space within a while loop, thus automating the analysis task within the modeling environment.

\subsection{Test alternative parameterizations}

Figure 9 demonstrates the following scenario. Assume we have nine different cloud physics schemes we wish to test in nine different runs. The easiest way to do this is to take advantage of Python's object-oriented inheritance capabilities, creating a new class NewQt $\mathrm{cm}$ that inherits everything from Qt $\mathrm{cm}$, and to which we add the additional cloud schemes (cloud0, cloud1, etc.). In the for loop in Fig. 9, we change the cloud model run list entry in the ' atm_physics1' run list to whatever the cloud model is at this point in the loop.

Of course, we could do the same thing by running the nine models separately, but this set-up makes it easy to do hypothesis testing between these nine models as the models are running. For instance, we can create a test by which we will choose which of the nine models to use: Within this framework, the selection of those models can be altered by changing a string. If the same task were implemented with shell scripts and makefiles, we would have to write our own selector routines (perhaps using file system functions) for selecting model(s) from amongst the possible executables. It is much easier to use Python's built-in string manipulation routines.

\section{Discussion and conclusions}

In the present work, an intermediate-level atmosphere model written in Fortran is wrapped with an object-oriented structure written in Python, which makes modern data abstraction utilities available to a model written in a traditional procedural language. The result is a model that can be used dynamically at run time, with the user able to change the order of subroutine execution at will, and able to analyze model results within the modeling environment.

This flexibility, however, potentially provides more than just convenience for the user. The qt $\mathrm{cm}$ package's run timeinteractive tools, and tools like them, can transform the traditional analysis sequence used in modeling studies into a 


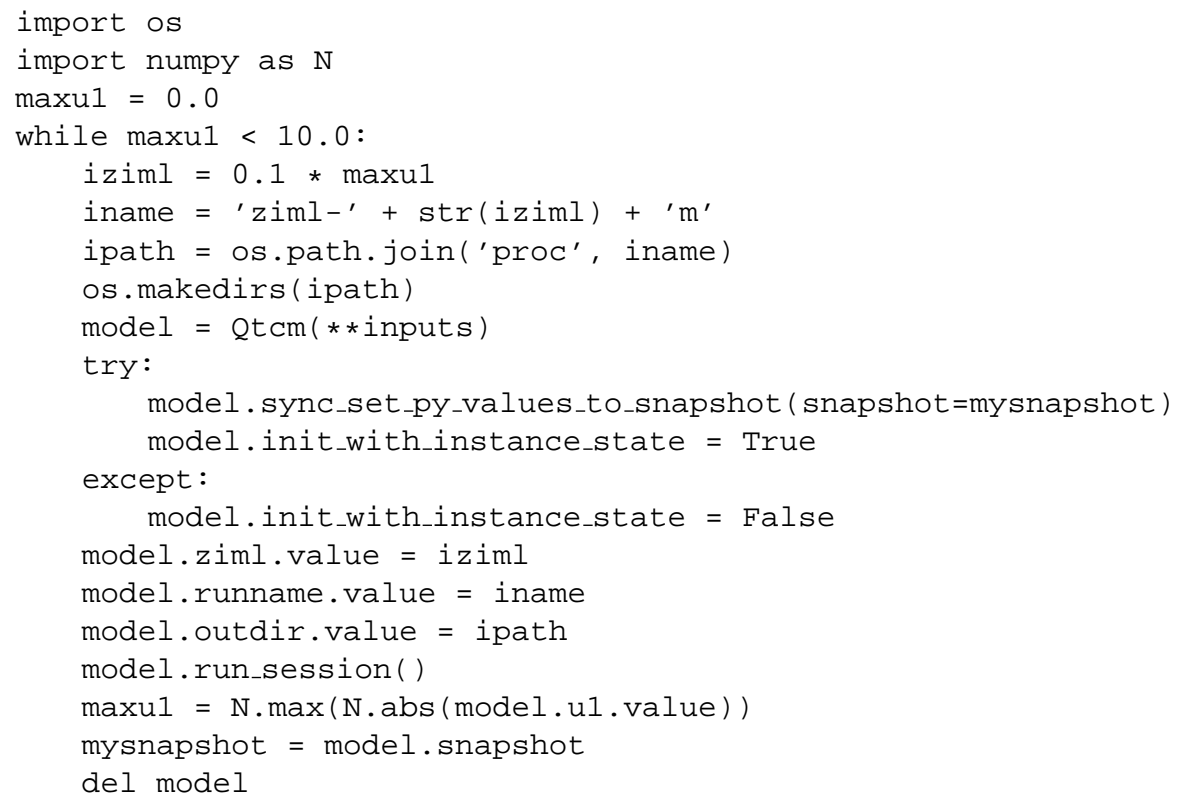

Fig. 8. Example of an exploration of the effects of different values of mixed-layer depth. The input s dictionary is initialized similarly as in Fig. 1.

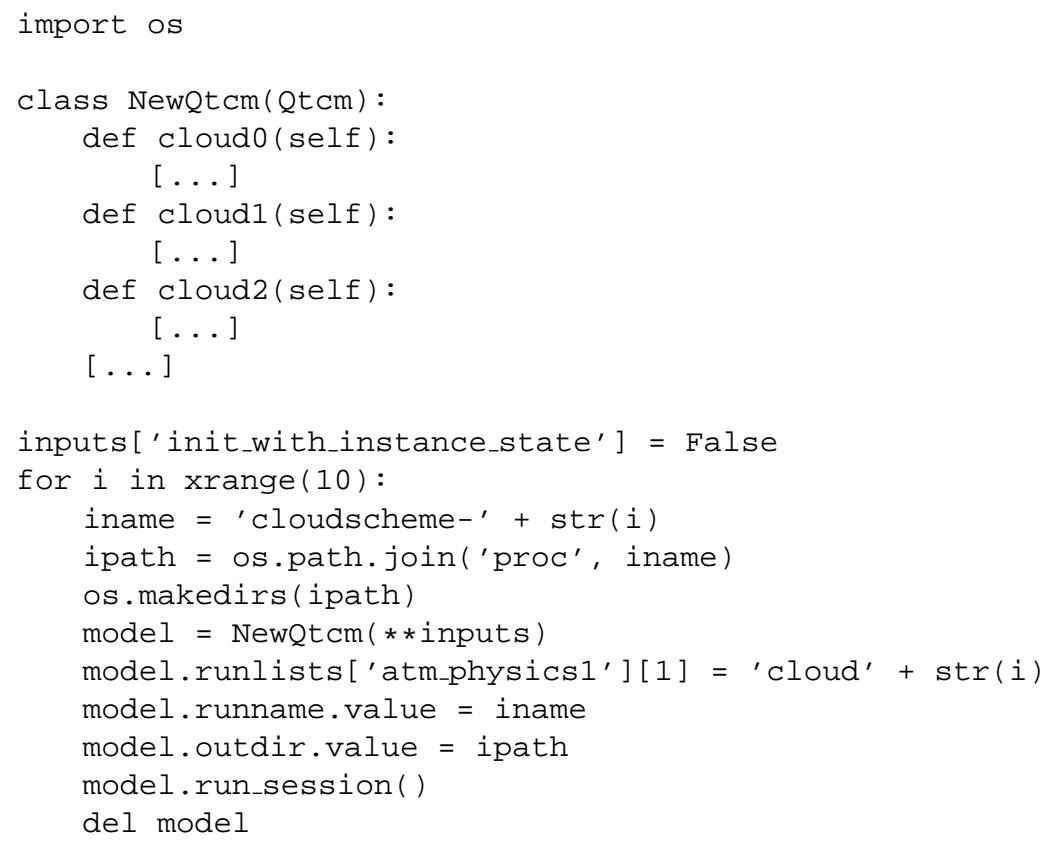

Fig. 9. Example of using inheritance in Python to explore the effects of multiple cloud physics schemes in multiple runs. The [ . . . ] denote the code of the different (hypothetical) cloud physics schemes. The inputs dictionary is defined similarly as in Fig. 1.

sequence with more capabilities. The traditional sequence begins with formulation of a hypothesis, then leads to implementing a test of the hypothesis in model code, making model runs using the coded test, and ends with analyzing the model results using various statistical and visualization packages (Fig. 10a). Some transitions between the various steps mainly make use of human input (e.g., from hypoth- esis to code), while others combine human reasoning with computational tools (e.g., we can mostly automate the transition from code to model runs through the use of makefiles combined with shell scripts). The feedback part of the cycle, where analysis of the results modifies the original hypothesis, usually requires human input. 
(a)

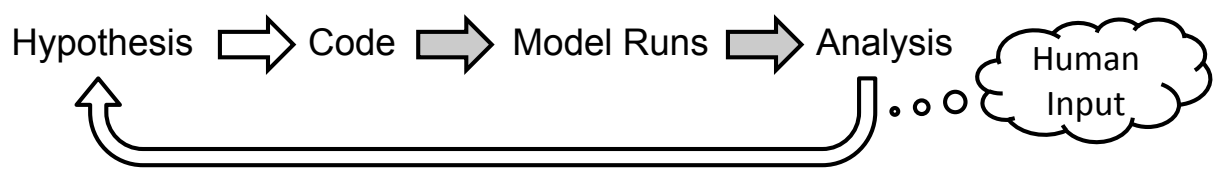

(b)

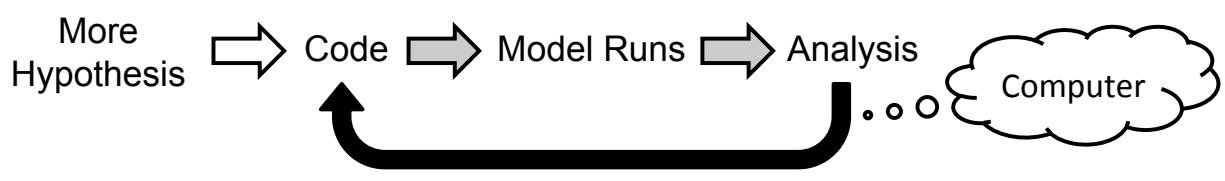

Fig. 10. Schematic of (a) the traditional analysis sequence used in modeling studies, and (b) the transformed analysis sequence using qt cm-like modeling tools. Outlined arrows with no fill represent mainly human input. Gray-filled arrows represent a mix of human and computer-controlled input. Completely filled (black)-arrows represent purely computer-controlled input.

In contrast, the tools provided by qt $\mathrm{cm}$ and similar packages open up the potential to automate substantially larger portions of the analysis sequence. Figure $10 \mathrm{~b}$ shows a schematic of how model analysis might be transformed. Instead of being limited to a few hypotheses, the transformed sequence makes additional types of hypotheses accessible without changing the complexity of the code required (see Sect. 4's examples as illustrations). Most importantly, the Fig. 10b sequence enables model output analysis to automatically control future model runs. Instead of requiring human intervention to determine future model runs, the computer can make that evaluation, and as a result, for the same complexity of code, we can more intelligently explore the problem's solution space.

Thus, though the use of mixed language programming environments for climate modeling has a modest cost in performance, these environments have the potential the pay back substantial dividends in code simplicity, reliability, and easeof-use. More importantly, such an environment, by providing a robust programming interface with capabilities traditional languages cannot easily support, gives researchers the tools to investigate previously inaccessible (or difficult to access) questions. The wrapping techniques illustrated in the present study for the Neelin-Zeng QTCM1 may be fruitfully deployed to other climate models, increasing their flexibility and scientific usefulness.

Acknowledgements. Thanks to David Neelin, Ning Zeng, Matthias Munnich, and the Climate Systems Interactions Group at UCLA for encouragement and help. Thanks to Alexis Zubrow, Christian Dieterich, Rodrigo Caballero, Michael Tobis, and Ray Pierrehumbert for Python help. Comments by reviewers Charles Doutriaux and Sebastien Denvil were very helpful. Early development of qt cm precursors was carried out at the University of Chicago Climate Systems Center, funded by the National Science Foundation (NSF) Information Technology Research
Program under grant ATM-0121028. Any opinions, findings and conclusions or recommendations expressed in this material are those of the author and do not necessarily reflect the views of the NSF. Trademarks in the present work are the property of their respective owners.

Edited by: O. Marti

\section{References}

Beazley, D. M.: SWIG 1.1 Users Manual, http://www.swig.org/ Doc1.1/HTML/Contents.html, 1997.

Edwards, P. N.: A brief history of atmospheric general circulation modeling, in: General Circulation Development, Past Present and Future: The Proceedings of a Symposium in Honor of Akio Arakawa, edited by: Randall, D. A., Academic Press, New York, 67-90, 2000.

Gushchina, D., Dewitte, B., and Illig, S.: Remote ENSO forcing versus local air-sea interaction in QTCM: A sensitivity study to intraseasonal variability, Adv. Geosci., 6, 289-297, 2006, http://www.adv-geosci.net/6/289/2006/.

Hunter, J. and Dale, D.: The Matplotlib User's Guide, http:// matplotlib.sourceforge.net/users_guide_0.98.1.pdf, 2007.

Johnson, R. A.: Object-oriented analysis and design - What does the research say?, J. Comput. Inform. Syst., 42, 11-15, 2002.

Lin, J. W.-B.: qtem User's Guide, http://www.johnny-lin.com/py pkgs/qtcm/doc/manual.pdf, 2008.

Lin, J. W.-B. and Neelin, J. D.: Influence of a stochastic moist convective parameterization on tropical climate variability, Geophys. Res. Lett., 27, 3691-3694, 2000.

Lin, J. W.-B. and Neelin, J. D.: Considerations for stochastic convective parameterization, J. Atmos. Sci., 59, 959-975, 2002.

Lin, J. W.-B., Neelin, J. D., and Zeng, N.: Maintenance of tropical intraseasonal variability: Impact of evaporation-wind feedback and midlatitude storms, J. Atmos. Sci., 57, 2793-2823, 2000.

Mesinger, F. and Arakawa, A.: Numerical Methods Used in Atmospheric Models, Vol. 1, GARP Publications Series No. 17, World Meteorological Organization, 1976. 
Neelin, J. D. and Zeng, N.: A quasi-equilibrium tropical circulation model - formulation, J. Atmos. Sci., 57, 1741-1766, 2000.

Neelin, J. D., Zeng, N., Chou, C., Lin, J., Su, H., Munnich, M., Hales, K., and Meyerson, J.: The Neelin-Zeng Quasi-Equilibrium Tropical Circulation Model (QTCM1), Version 2.3, UCLA Department of Atmospheric Sciences, Los Angeles, http://www.atmos.ucla.edu/ csi/qtcm_man/v2.3/qtcm_ manv2.3.pdf, 2002.

Oliphant, T. E.: Python for scientific computing, Comput. Sci. Eng., 9, 10-20, 2007.

PCMDI: Climate Data Analysis Tools, http://cdat.sf.net, 2006.

Pennington, N., Lee, A. Y., and Rehder, B.: Cognitive activities and levels of abstraction in procedural and object-oriented design, Hum.-Comput. Interact., 10, 171-226, 1995.

Peterson, P.: F2PY Users Guide and Reference Manual, http://cens. ioc.ee/projects/f2py2e/usersguide/index.html, 2005.
PyCCSM: pyccsm: A Python version of the CCSM coupler, http: //code.google.com/p/pyccsm/, 2008.

Unidata: The NetCDF Tutorial, Boulder, CO, http://www.unidata. ucar.edu/software/netcdf/docs/netcdf-tutorial.html, 2007.

van der Walt, S.: Documentation: NumPy and SciPy, http://www. scipy.org/Documentation, 2008.

van Rossum, G.: Python Tutorial: Release 2.5.2, Python Software Foundation, http://www.python.org/doc/2.5.2/tut/tut.html, 2008.

Zeng, N., Neelin, J. D., Lau, K.-M., and Tucker, C. J.: Enhancement of interdecadal climate variability in the Sahel by vegetation interaction, Science, 286, 1537-1540, 1999.

Zeng, N., Neelin, J. D., and Chou, C.: A quasi-equilibrium tropical circulation model-implementation and simulation, J. Atmos. Sci., 57, 1767-1796, 2000. 